\title{
BMJ Open Efficacy of quadruple regimen with polaprezinc for gastric Helicobacter pylori infection eradication: protocol for a single-centre, single-blind, non- inferiority, randomised clinical trial
}

\author{
Dingkun Wu (D) , ${ }^{1,2}$ Zhen Sun, ${ }^{3}$ Tingyuan Li, ${ }^{2}$ Qinwen Tan, ${ }^{2,4}$ Yue Sun, ${ }^{2}$ \\ Tingting Chen, ${ }^{5}$ Yujing Liu, ${ }^{6}$ Jun $\mathrm{Li}^{7}{ }^{7}$ Haidong Jiang, ${ }^{7}$ Zhiqiang Yuan, ${ }^{7}$ \\ Yuqian Zhao (1) , ${ }^{2}$ Wen Chen ${ }^{2,6}$
}

To cite: Wu D, Sun Z, Li T, et al. Efficacy of quadruple regimen with polaprezinc for gastric Helicobacter pylori infection eradication: protocol for a single-centre, single-blind, non-inferiority, randomised clinical trial. BMJ Open 2020;10:e037182. doi:10.1136/ bmjopen-2020-037182

- Prepublication history and additional material for this paper is available online. To view these files, please visit the journal online (http://dx.doi.org/10. 1136/bmjopen-2020-037182)

Received 22 January 2020 Revised 11 September 2020 Accepted 22 September 2020

A Check for updates

(c) Author(s) (or their employer(s)) 2020. Re-use permitted under CC BY-NC. No commercial re-use. See rights and permissions. Published by BMJ.

For numbered affiliations see end of article.

\section{Correspondence to}

Dr Yuqian Zhao;

gw_zhaoyuqian@126.com and

Dr Wen Chen;

chenwen@cicams.ac.cn

\section{ABSTRACT}

Introduction Helicobacter pylori (H. pylori) is the most well-known risk factor for gastric cancer. At present, H. pylori shows varying levels of resistance to different treatments, leading to a lower rate of $H$. pylori eradication. The aim of this study is to evaluate the efficacy of polaprezinc-containing quadruple therapy (PQT) for the eradication of $H$. pylori infection and, thus, to provide more evidence to inform the clinical treatment of $H$. pylori infection in China.

Methods and analysis This is a single-centre, singleblind, non-inferiority, randomised controlled trial, enrolling 158 patients with $H$. pylori infection. Patients are randomised (1:1) to the two groups for a 14-day therapy. Treatment group: PQT (esomeprazole $20 \mathrm{mg}$, amoxicillin $1 \mathrm{~g}$, clarithromycin $500 \mathrm{mg}$, polaprezinc $75 \mathrm{mg}$ ) two times per day; control group: bismuth-containing quadruple therapy (esomeprazole $20 \mathrm{mg}$, amoxicillin $1 \mathrm{~g}$, clarithromycin $500 \mathrm{mg}$, bismuth potassium citrate $220 \mathrm{mg}$ ) two times per day. The primary outcome is the rate of $H$. pylori eradication. Secondary outcomes are the incidence of adverse events and the gastrointestinal microbiota distribution. The $16 \mathrm{~S}$ ribosomal RNA (16S rRNA) next-generation sequencing (NGS) is used to evaluate the effect of two different therapies on the distribution of the gastrointestinal microbiota.

Ethics and dissemination This study was approved by the Ethics Committee of Sichuan Cancer Center \& Hospital (No. SCCHEC-02-2019-015). Any amendment to the research protocol will be submitted for ethical approval. All participants must provide informed consent. On completion, the results of the study will be published in the appropriate peer-reviewed journal.

Trial registration number ChiCTR1900025800; preresults.

\section{INTRODUCTION}

Helicobacter pylori (H. Pylori) is a multiflagellum, microaerobic Gram-negative, bacillus, that is specifically colonised in the gastric epithelium. ${ }^{1}$ It is estimated that about

\section{Strengths and limitations of this study}

- The study design (randomised controlled trial, RCT) is the gold standard of clinical evidence.

- This is the first RCT to compare the efficacy of polaprezinc-containing quadruple therapy and bismuth-containing quadruple therapy on $\mathrm{H}$. pylori eradication.

- The results of this study could provide more evidence to inform the clinical treatment of $H$. pylori infection in China.

- This is the first prospective study with nextgeneration sequence detecting strategy in Chinese mainland to explore the possible drug-resistance mechanism of $H$. pylori.

- The single-centre clinical trial may not represent the H. pylori eradication rate of general population.

$50 \%$ of the world's population is infected with $H$. pylori, ${ }^{2}{ }^{3}$ but the infection rate for H. pylori varies widely by population, age, geographic region, race, socioeconomic status, health status, population density and eating habits. ${ }^{23}$ H. pylori is one of the most important pathogenic factors of gastrointestinal diseases such as chronic gastritis, peptic ulcer, gastric adenocarcinoma and gastric mucosa - associated lymphoid tissue (MALT) lymphoma. ${ }^{45}$ Moreover, H. pylori infection is correlated with a 1 -fold to 10 -fold increase in the risk of gastric or duodenal ulcer and with $0.1 \%-3 \%$ risk of developing gastric cancer. ${ }^{6}$ The eradication of $H$. pylori is an important method for the treatment of digestive tract ulceration and chronic gastritis and for the prevention of gastric cancer.

At present, a combination of therapy is recommended for the treatment of $H$. pylori infection in clinical practice as well as for the 
treatment of gastritis and digestive ulcer caused by $H$. pylori infection. Among available treatments, the use of bismuth, in combination with antibiotics, such as metronidazole, amoxicillin, clarithromycin, azithromycin, levofloxacin, chloramphenicol and tetracycline, has been widely recommended. Polaprezinc, a chelating agent of L-carnosine and zinc, can block the colonisation of $H$. pylori by inhibiting its activity pharmacologically and the associated inflammatory chain reaction and removing urea, enzymes, monochloramines to achieve the $H$. pylori eradication. Polaprezinc combination with lansoprazole, amoxicillin and clarithromycin can increase the $H$. pylori eradication rate from $24 / 31\left(77.4 \%\right.$, triple therapy alone) to $33 / 35(94.3 \%){ }^{7}$ However, the efficacy of $H$. pylori infection treatment in high-risk population in China is needed to be established.

With the frequent use of antibiotics, the rate of antibiotic resistance also showed an upward trend. Studies reported on the resistances of $H$. pylori to various antibiotics, which lead to lower $H$. pylori eradication rates. A large number of studies outside China have been conducted to detect the drug-resistance sites of $H$. pylori, with the plausible mechanisms leading to resistance for many antibiotics having been defined. ${ }^{8-12}$ Moreover, the use of drugs to treat $H$. pylori infection has been associated with significantly different degrees of overgrowth of the gastric microbiota. ${ }^{13}$ Compared with patients with gastritis, the diversity of gastric microbiota among patients with gastric cancer is significantly lower, with the abundance of Helicobacter species being specifically decreased, while the abundance of other species is increased. ${ }^{14}$ The effect of different treatment options for treatment of $H$. pylori infection on the distribution of the other microbiota in the gastrointestinal is unknown.

The 16S ribosomal RNA (16S rRNA) next-generation sequencing(NGS) technology is a powerful tool for microbial detection and classification. The $16 \mathrm{~S}$ rRNA sequence information is obtained by comparing the gene sequences of the 16S rRNA gene fragment of various organisms, with the sequence information obtained by cloning, sequencing or enzyme cutting or probe hybridisation. The 16S rRNA sequence information is then compared with the data in the $16 \mathrm{~S}$ rRNA database for identification and is not affected by antibiotics. It can identify dead bacteria and microorganisms that currently cannot be cultivated artificially as well as identify new types of microorganisms that play an important role in intestinal microbiota detection. ${ }^{15} 16$

The aim of our study is to evaluate the efficacy of polaprezinc-containing quadruple therapy (PQT) compared with the traditionally bismuth-containing quadruple therapy (BQT) for $H$. pylori eradication and, thus, to provide evidence for clinical treatment of $H$. pylori infection in Chinese population.

\section{METHOD AND ANALYSIS Design}

This protocol is a single-centre, single-blind, noninferiority, randomised clinical trial, in which 158 patients with $H$. pylori infection will be enrolled. Patients will be randomly assigned into one of the study groups in a 1:1 allocation ratio (figure 1). Patients are required to come to hospital on day 7 after taking drugs for receiving nextcycle drugs and follow-up the adverse events (AEs). Phone follow-up is used to monitor AEs on day 14 after taking drugs. All patients will be called back within week 4-8 after the end of therapy. This study follows the Consolidated Standards of Reporting Trials guidelines.

Measured outcomes (and associated processes) are summarised in table 1 . The $16 \mathrm{~S}$ rRNA will be performed to sequence the gastrointestinal microbiota in the collected samples.

This study will be conducted at Yanting County Cancer Hospital. It began in December 2019 and is projected to take 1 year to complete.

\section{Objectives}

The three objectives of the study are as follows. The first is to evaluate the efficacy and safety of PQT for eradication of $H$. pylori infection. The second is to detect the antibiotic drug-resistance mutation sites of $H$. pylori and explore the association between the therapy and the drug-resistance sites. The third is to compare the distribution of gastrointestinal microbiota, before and after the therapy and between the different therapies.

\section{Inclusion criteria}

The enrolled patients should be 18-65 years of age, residents who participated in early screening for upper gastrointestinal cancer and the outpatients at Yanting County Cancer Hospital, with a positive primary ${ }^{14} \mathrm{C}$ urea breath test $\left({ }^{14} \mathrm{C}-\mathrm{UBT}\right)$ for $H$. pylori infection, with no evidence of gastric cancer or other severe gastric disease identified on gastroscopy and should provide written informed consent.

\section{Exclusion criteria}

Potential participants will be screened on the following exclusion criteria: prior antibiotics or bismuth therapy within 4 weeks of enrolment; prior proton pump inhibitor (PPI) therapy within 2 weeks of enrolment; pregnancy or lactation in women; history of cardiovascular and other severe diseases; participation in other clinical studies within 3 months; unable to follow the study procedures (eg, due to mental illness or severe neurosis); history of allergy to medications used and any contraindication to gastroscopy.

\section{Criteria for termination in the study}

The following criteria will support termination of a patient's participation in the study: deterioration of health status or severe complications; severe adverse effect on the drug used that cannot be tolerated by the patient; other conditions that would affect the measurement of the outcomes; pregnancy during the treatment and lost to follow-up. 


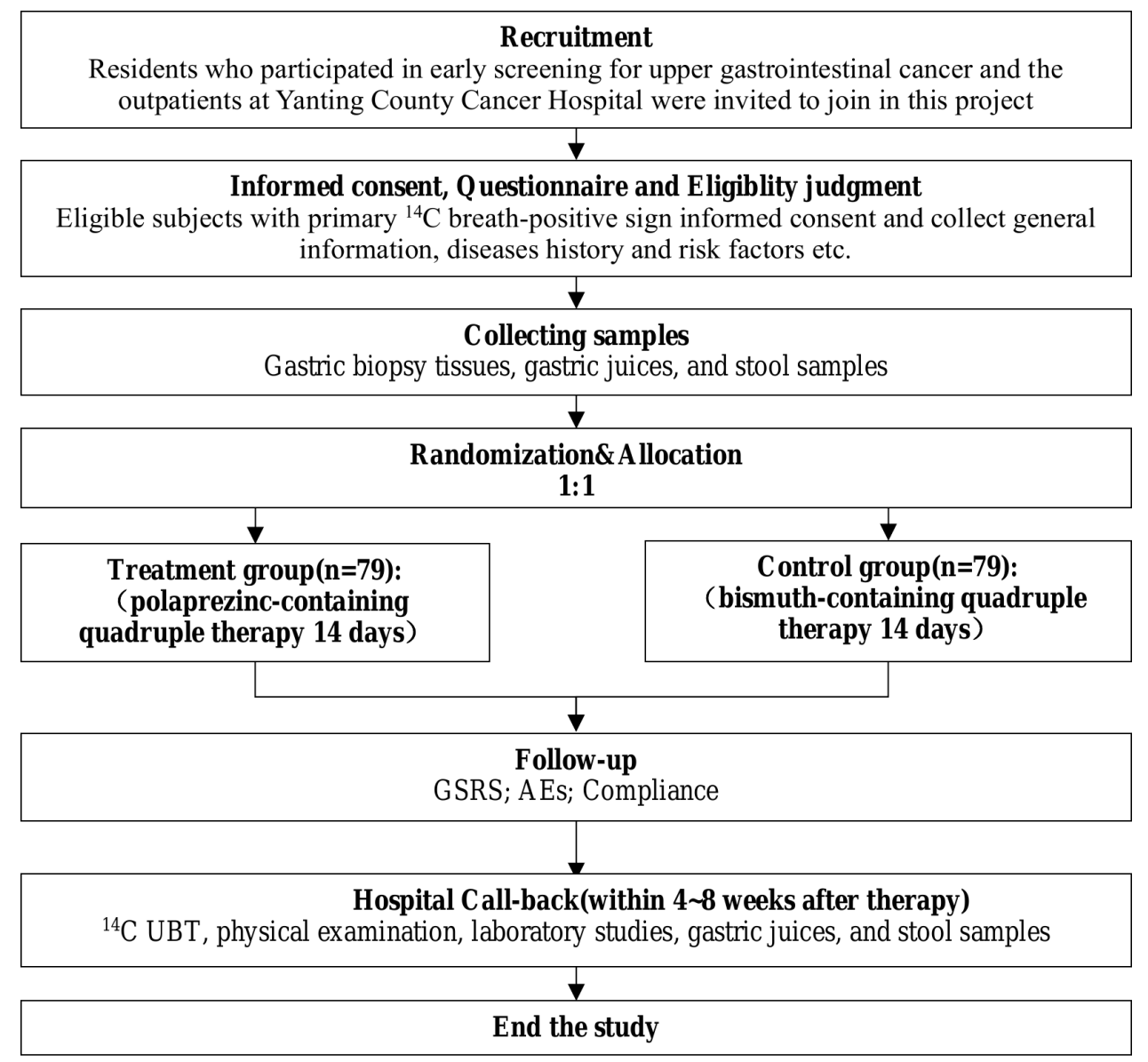

Figure 1 Flowchart of the clinical study. treatment group: esomeprazole, amoxicillin, clarithromycin and polaprezinc. Control group: esomeprazole, amoxicillin, clarithromycin and bismuth potassium citrate. ${ }^{14} \mathrm{C}$ UBT, ${ }^{14} \mathrm{C}$-urea breath test; AEs, adverse eventsGSRS, gastrointestinal symptom rating scale.

\section{Allocation and blinding}

Patients will be randomly allocated to the two groups of the study, namely, the treatment group (PQT) and the control group $(\mathrm{BQT})$, by an independent researcher using a computer-generated random sequence, with a 1:1 allocation ratio. The drugs are packaged in identical boxes, ensuring that the drugs cannot be identified on packaging appearance. The drugs are controlled by an independent drug administrator and the doctor assigned the drugs to the participants strictly according to the protocol. All patients in the study will be blinded to the assignment.

\section{Interventions}

All enrolled patients will complete physical examination and laboratory tests, including complete blood count, blood chemistry and urine tests, electrocardiogram (ECG) and gastroscope. Stools, gastric juices and biopsy tissues will be collected before therapy. Patients with a confirmed $H$. pylori infection, who meet the inclusion criteria, will be randomly assigned to the two groups of the study. As per the sample size calculation described below, 79 patients will be enrolled into each group. Patients in the treatment group will receive a 14-day PQT (esomeprazole $20 \mathrm{mg}$, amoxicillin $1 \mathrm{~g}$, clarithromycin $500 \mathrm{mg}$, polaprezinc $75 \mathrm{mg}$ ) two times per day. Patients in the control group will receive a 14-day BQT (esomeprazole $20 \mathrm{mg}$, amoxicillin $1 \mathrm{~g}$, clarithromycin $500 \mathrm{mg}$, bismuth potassium citrate $220 \mathrm{mg}$ ) two times per day.

\section{Outcomes}

The primary outcome of this study is the rate of $H$. pylori eradication, 4 weeks after the end of therapy, as measured by a ${ }^{14} \mathrm{C}$-UBT. The secondary outcomes are the incidence of AEs and the gastrointestinal microbiota distribution before and after the therapy and between the different therapies. 
Table 1 Project flow and follow-up items.

\begin{tabular}{|c|c|c|c|c|}
\hline \multirow[b]{2}{*}{ Project } & \multirow{2}{*}{$\begin{array}{l}\text { Before treatment/ } \\
\text { baseline } \\
0 \text { week ( }-5 \text { to }-1 \text { days) }\end{array}$} & \multicolumn{2}{|c|}{$\begin{array}{l}\text { Period of treatment/intervention } \\
\text { and follow-up ( } 14 \text { days) }\end{array}$} & \multirow{2}{*}{$\begin{array}{l}\text { After the treatment/ } \\
\text { call-back } \\
\text { Within 4-8 weeks }\end{array}$} \\
\hline & & 1 week/day 7 & 2 weeks/day 14 & \\
\hline Informed consent & $x$ & & & \\
\hline Inclusion and exclusion criteria & $x$ & & & \\
\hline Randomisation & $x$ & & & \\
\hline Blood routine test & $x$ & & & $x$ \\
\hline Urine routine test & $x$ & & & $x$ \\
\hline Renal function test & $x$ & & & $x$ \\
\hline Stool $H$. pylori antigen & $x$ & & & $x$ \\
\hline Specimens & $x$ & & & $x$ \\
\hline Treatment and follow-up & & $x$ & $x$ & $x$ \\
\hline Concomitant medications & $x$ & $x$ & $x$ & $x$ \\
\hline AEs & & $x$ & $x$ & $x$ \\
\hline Surplus medicines recycling & & & $x$ & \\
\hline
\end{tabular}

The ' $x$ ' is the item or operation content that must be performed in the project.

${ }^{14} \mathrm{C}$ UBT, ${ }^{14} \mathrm{C}$-urea breath test; AEs, adverse events.

\section{Follow-up}

Self-reported severity of gastrointestinal symptoms is assessed on day 7 and 14 after taking drugs. The Gastrointestinal Symptom Rating Scale (GSRS) has 15 items included in five symptom clusters depicting reflux, abdominal pain, indigestion, diarrhoea and constipation, its symptoms are graded on a 7-point Likert scale (1 means absence of symptoms, 7 means with much trouble symptoms). The GSRS was found to be good, with acceptable reliability and validity. ${ }^{17}$ Self-reported symptom severity is obtained by patient interviews on day 7 and day 14 after taking drugs. Patients are required to come to hospital on day 7 after taking drugs for receiving next-cycle drugs and follow-up the AEs. Phone follow-up is used to monitor AEs on day 14 after taking drugs. All AEs and serious AEs are recorded and will be submitted to the Medical Ethics Committee of Sichuan Cancer Center \& Hospital(SCC).

${ }^{14} \mathrm{C}$-UBT is carried out for confirmation of $H$. pylori eradication. $^{18}$ Physical examination and laboratory studies (including complete blood count, blood chemistries, urine test, ECG and gastroscopy) will be repeated 4-8 weeks after the end of therapy. Meanwhile, stools, gastric juices will be collected.

\section{Sample size estimation}

As appropriate for a non-inferiority study, the calculation of the sample size was based on the primary outcome, the eradication rate of $H$. pylori. A previous single-centre, small sample size study conducted in Japan determined that polaprezinc combined with triple therapy increased the $H$. pylori eradication rate from $77.4 \%$ to $94.3 \%{ }^{7}$ Another randomised, open-label, non-inferiority, phase 3 study reported an $H$. pylori eradication rate of $80 \%$ when treated using bismuth subcitrate potassium, metronidazole and tetracycline, in combination with omeprazole, as a quadruple therapy. ${ }^{19}$ Based on these data, we calculated that 63 participants were required in each group for a one-sided $\alpha$ value of 0.025 and power of $80 \%$. A high proportion is better, control group proportion of $80 \%$ and the non-inferiority margin of $-20 \%$ are set to identify group difference in the rate of $H$. pylori eradication. Considering a drop out rate of $20 \%$, a sample of 79 patients in each group is required. The sample size analysis was performed using PASS (V.15.0; NCSS, LLC, USA).

\section{Patient and public involvement}

No patients or members of the public participated in the conception of our study. On completion, however, the results of the study will be published in the appropriate journal.

\section{Data collection and management}

A project-specific data entry group will be established at SCC before the initiation of the study. Two individuals are responsible for the data entry and double check the accuracy of the data entered. Data will be entered in 
an encrypted ACCESS database, developed by the data administrator at SCC, who is responsible for monitoring the data.

Access to the original case report form (CRF) and database is restricted to the data entry personnel, investigators and other relevant researchers authorised by the principal investigator.

\section{Data analysis}

All statistical analyses are performed by the statistician at SCC using SPSS (V.17.0; SPSS, Chicago, Illinois, USA). Intention-to-treat (ITT) and per-protocol (PP) analyses are used to evaluate the primary outcome of $H$. pylori eradication. The ITT population included patients who meet the criteria, randomised, take at least one dose of drugs after enrolled. Missing observations are accounted for using the predictive mean matching (PMM) method. The PP population included patients who complete the designated therapy of this protocol. Categorical variables are compared using $\chi^{2}$ test or Fisher exact test. Continuous data are compared using Student's t-test or Wilcoxon rank test. The primary analysis (evaluating non-inferiority of the PQT) is assessed through hypothesis testing and derivation of a one-sided $95 \%$ CIs. P values $<0.05$ were considered statistically significant.

The following processes are applied to the microbiota distribution of the secondary outcome data: quantitative insights into microbial ecology ${ }^{20}$ (QIIME2, https:// qiime2.org/) is used to clean and assemble the original offline data. The amplicon sequence variants (ASVs) are assigned to taxa (domain, kingdom, phylum, class, order, family, genus and species) by matching to SILVA database V138. The alpha diversity of gastrointestinal microbiota is based on Shannon's diversity index, observed ASVs, Faith's phylogenetic diversity and Pielou's evenness. Microbiota statistical analyses are performed using R (V.3.6.1 platform; https://www.r-project.org/). Principal coordinate analysis $(\mathrm{PCoA})$ is performed to represent the differences of the beta diversity based on Bray-Curtis, Jaccard distances, weighted unifrac distance and unweighted unifrac distance. The composition of microbiota is compared between different groups, and the different taxa are identified based on linear discriminant analysis (LDA) effect size(LEfSe). Adonis function in $R$ vegan is used to quantify the impact of host lifestyle on microbiome community.

\section{Specimen preservation}

Special specimen administrators are designated, for this study, at SCC and the study site to ensure the unified storage of specimens and the management of refrigerator keys. The specimen administrator sorts samples according to their ID number in a sequence. The specimens will be transferred to the SCC in drikold and stored at a temperature of $-80^{\circ} \mathrm{C}$.

\section{Privacy measures for patient data}

All specimens and forms collected as part of this study are coded using unique patient identifiers; electronic data are stored in an encrypted database on a passwordprotected platform, which can only be identified by authorised research personnel. As well, patient records will be accessed only as per the regulations of the Ethics Research Committee at the SCC, and only when necessary. To the extent permitted by applicable laws and/or regulations, any records relating to patient identification are confidential and will not be made public. Patients or their legal representatives are notified in a timely manner if new information arises that may affect a patient's continued participation in the study.

\section{Ethics and dissemination}

This study was approved by the Ethics Committee of Sichuan Cancer Center \& Hospital (No. SCCHEC-022019-015). Any amendment to the research protocol will be submitted for ethical approval. All participants must provide written informed consent (online supplemental material 1). On completion, the results of the study will be published in the appropriate peer-reviewed journal.

\section{Author affiliations}

${ }^{1}$ School of Medicine, University of Electronic Science and Technology of China, Chengdu, China

${ }^{2}$ Center for Cancer Prevention Research, Sichuan Cancer Hospital \& Institute, Sichuan Cancer Center, School of Medicine, University of Electronic Science and Technology of China, Chengdu, China

${ }^{3}$ Department of Gastroenterology, Jilin People's Hospital, Jilin, China

${ }^{4}$ Chongqing Medical University, Chongqing, China

${ }^{5}$ West China School of Public Health/West China Fourth Hospital, Sichuan University, Chengdu, China

${ }^{6}$ Department of Cancer Epidemiology, National Cancer Center/National Clinical Research Center for Cancer/Cancer Hospital, Chinese Academy of Medical Sciences and Peking Union Medical College, Beijing, China

${ }^{7}$ Cancer Prevention and Treatment Office, Yanting Cancer Hospital, Mianyang, China

Acknowledgements We thank all the participants and their advisors involving in this study, especially the health providers in Yanting Cancer Hospital.

Contributors DW, TL, YZ and WC contributed to the study design. ZY, JL and $\mathrm{HJ}$ contributed to the data collection. DW, ZS, TL, QT, TC, YL, YZ, WC and YS supervised the field study. DW, ZS and TL drafted the initial manuscript. All authors have reviewed and revised the final manuscript.

Funding This study was supported by Chinese Academy of Medical Sciences Innovation Fund for Medical Sciences (CIFMS) (2016-I2M-3-001).

Competing interests None declared.

Patient consent for publication Obtained.

Provenance and peer review Not commissioned; externally peer reviewed.

Supplemental material This content has been supplied by the author(s). It has not been vetted by BMJ Publishing Group Limited (BMJ) and may not have been peer-reviewed. Any opinions or recommendations discussed are solely those of the author(s) and are not endorsed by BMJ. BMJ disclaims all liability and responsibility arising from any reliance placed on the content. Where the content includes any translated material, BMJ does not warrant the accuracy and reliability of the translations (including but not limited to local regulations, clinical guidelines, terminology, drug names and drug dosages), and is not responsible for any error and/or omissions arising from translation and adaptation or otherwise.

Open access This is an open access article distributed in accordance with the Creative Commons Attribution Non Commercial (CC BY-NC 4.0) license, which permits others to distribute, remix, adapt, build upon this work non-commercially, and license their derivative works on different terms, provided the original work is properly cited, appropriate credit is given, any changes made indicated, and the use is non-commercial. See: http://creativecommons.org/licenses/by-nc/4.0/. 


\section{ORCID iDs}

Dingkun Wu http://orcid.org/0000-0003-1600-9571

Yuqian Zhao http://orcid.org/0000-0002-2736-4766

\section{REFERENCES}

1 Warren JR, Marshall B. Unidentified curved bacilli on gastric epithelium in active chronic gastritis. Lancet 1983;1:1273-5.

2 Hooi JKY, Lai WY, Ng WK, et al. Global prevalence of Helicobacter pylori infection: systematic review and meta-analysis. Gastroenterology 2017;153:420-9.

3 Zamani M, Ebrahimtabar F, Zamani V, et al. Systematic review with meta-analysis: the worldwide prevalence of Helicobacter pylori infection. Aliment Pharmacol Ther 2018;47:868-76.

4 Suerbaum S, Michetti P. Helicobacter pylori infection. N Engl J Med 2002;347:1175-86.

5 Atherton JC. The pathogenesis of Helicobacter pylori-induced gastro-duodenal diseases. Annu Rev Pathol 2006;1:63-96.

6 McColl KEL. Clinical practice. Helicobacter pylori infection. N Engl J Med 2010;362:1597-604.

7 Kashimura H, Suzuki K, Hassan M, et al. Polaprezinc, a mucosal protective agent, in combination with lansoprazole, amoxycillin and clarithromycin increases the cure rate of Helicobacter pylori infection. Aliment Pharmacol Ther 1999;13:483-7.

8 Goodwin A, Kersulyte D, Sisson G, et al. Metronidazole resistance in Helicobacter pylori is due to null mutations in a gene ( $\mathrm{rdxA})$ that encodes an oxygen-insensitive NADPH nitroreductase. Mol Microbiol 1998;28:383-93.

9 Debets-Ossenkopp YJ, Pot RG, van Westerloo DJ, et al. Insertion of mini-IS605 and deletion of adjacent sequences in the nitroreductase ( $r d x A)$ gene cause metronidazole resistance in Helicobacter pylori NCTC11637. Antimicrob Agents Chemother 1999;43:2657-62.

10 Rimbara E, Noguchi N, Kawai T, et al. Fluoroquinolone resistance in Helicobacter pylori: role of mutations at position 87 and 91 of GyrA on the level of resistance and identification of a resistance conferring mutation in GyrB. Helicobacter 2012;17:36-42.

11 Hwang TJ, Kim N, Kim HB, et al. Change in antibiotic resistance of Helicobacter pylori strains and the effect of $\mathrm{A} 2143 \mathrm{G}$ point mutation of 23S rRNA on the eradication of $H$. pylori in a single center of Korea. J Clin Gastroenterol 2010;44:1-43.

12 Binh TT, Shiota S, Suzuki R, et al. Discovery of novel mutations for clarithromycin resistance in Helicobacter pylori by using nextgeneration sequencing. J Antimicrob Chemother 2014;69:1796-803.

13 Guo Y, Zhang Y, Gerhard M, et al. Effect of Helicobacter pylori on gastrointestinal microbiota: a population-based study in Linqu, a high-risk area of gastric cancer. Gut 2020;69:gutjnl-2019-319696.

14 Ferreira RM, Pereira-Marques J, Pinto-Ribeiro I, et al. Gastric microbial community profiling reveals a dysbiotic cancer-associated microbiota. Gut 2018;67:226-36.

15 Guo M-wei, Liu F-ming, Zhou W. Research progress of $16 \mathrm{~S}$ rRNA sequencing technology in detection of intestinal flora. Laboratory Sci 2019;22:1-4.

16 Almonacid DE, Kraal L, Ossandon FJ, et al. 16S rRNA gene sequencing and healthy reference ranges for 28 clinically relevant microbial taxa from the human gut microbiome. PLoS One 2017;12:e0176555.

17 Kulich KR, Madisch A, Pacini F, et al. Reliability and validity of the gastrointestinal symptom rating scale (GSRS) and quality of life in reflux and dyspepsia (QOLRAD) questionnaire in dyspepsia: a sixcountry study. Health Qual Life Outcomes 2008;6:12.

18 Malfertheiner P, Megraud F, O'Morain CA, et al. Management of Helicobacter pylori infection-the Maastricht V/Florence consensus report. Gut 2017;66:6-30.

19 Malfertheiner P, Bazzoli F, Delchier J-C, et al. Helicobacter pylori eradication with a capsule containing bismuth subcitrate potassium, metronidazole, and tetracycline given with omeprazole versus clarithromycin-based triple therapy: a randomised, open-label, noninferiority, phase 3 trial. Lancet 2011;377:905-13.

20 Bolyen E, Rideout JR, Dillon MR, et al. Reproducible, interactive, scalable and extensible microbiome data science using QIIME 2. Nat Biotechnol 2019;37:852-7. 